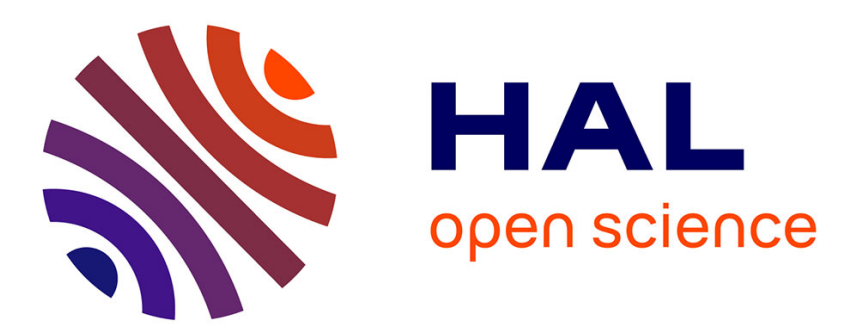

\title{
A conservative method for the simulation of the isothermal Euler system with the van-der-Waals equation of state
}

\author{
Vincent Perrier
}

\section{- To cite this version:}

Vincent Perrier. A conservative method for the simulation of the isothermal Euler system with the van-der-Waals equation of state. Journal of Scientific Computing, 2011, 48 (1-3), pp.296-303. 10.1007/s10915-010-9415-9 . inria-00633917

\section{HAL Id: inria-00633917 https://hal.inria.fr/inria-00633917}

Submitted on 19 Oct 2011

HAL is a multi-disciplinary open access archive for the deposit and dissemination of scientific research documents, whether they are published or not. The documents may come from teaching and research institutions in France or abroad, or from public or private research centers.
L'archive ouverte pluridisciplinaire HAL, est destinée au dépôt et à la diffusion de documents scientifiques de niveau recherche, publiés ou non, émanant des établissements d'enseignement et de recherche français ou étrangers, des laboratoires publics ou privés. 


\title{
A conservative method for the simulation of the isothermal Euler system with the van-der-Waals equation of state.
}

\author{
Vincent Perrier \\ INRIA Bordeaux Sud-Ouest \\ Laboratoire de Mathématiques et de leurs Applications \\ Avenue de l'Université, 64000 Pau
}

18 August 2010

\begin{abstract}
In this article, we are interested in the simulation of phase transition in compressible flows, with the isothermal Euler system, closed by the van-der-Waals model. We formulate the problem as an hyperbolic system, with a source term located at the interface between liquid and vapour. The numerical scheme is based on [1, 5]. Compared with previous discretizations of the van-der-Waals system, the novelty of this algorithm is that it is fully conservative. Its Godunov-type formulation allows an easy implementation on multidimensional unstructured meshes.
\end{abstract}

\section{Introduction}

Simulation of phase transition in compressible media is a difficult task, at least for two reasons:

- Which model to use for modelling phase transition is still an open question.

- The compressible nature of the flow means that the underlying system is hyperbolic. The theory for such systems is not complete [8]: for example, the functional space in which the Cauchy problem is well-posed is unknown, so that the analysis of schemes is hard.

In this paper, we will concentrate on the discretization of the isothermal Euler system. The system of equations reads

$$
\left\{\begin{array}{l}
\partial_{t} \rho+\operatorname{div}(\rho \mathbf{u})=0 \\
\partial_{t}(\rho \mathbf{u})+\operatorname{div}(\rho \mathbf{u} \otimes \mathbf{u}+p \mathcal{I})=0
\end{array}\right.
$$


which describes the conservation of the density $\rho$ and momentum $\rho \mathbf{u}$. When needed, (1) will be noted in the following simplified form

$$
\partial_{t} \mathbf{U}+\nabla \cdot F(\mathbf{U})=0
$$

The thermodynamic variables $\rho$ and $p$ are linked by the adimensioned van-der-Waals equation of state:

$$
p(\rho)=\frac{8 T \rho}{3-\rho}-3 \rho^{2}
$$

where $T$, the adimensioned temperature, is fixed, and the density is such that $0<\rho<3$. Such a model is widely used in the mathematical community for describing phase transition $[9,6]$.

This article is organised as follows: in Section 2, we make a brief review on the problems raised by the system, and the methods used for circumvent these problems. In Section 3, we reformulate the system as a multiphase system with a source term. An essential step for performing a finite volume approximation of an hyperbolic system is to solve the Riemann problem. How to solve this problem will be explained in Section 4. In Section 5, we expose a numerical scheme, which is tested in Section 6.

\section{Problems raised by the system}

The Jacobian of the system (1) in dimension 1 is

$$
\left(\begin{array}{cc}
0 & 1 \\
\left(p^{\prime}(\rho)-u^{2}\right) & 2 u
\end{array}\right)
$$

and its characteristic polynomial is $(X-u)^{2}-p^{\prime}(\rho)=0$. It admits real eigenvalues provided we have $p^{\prime}(\rho)>0$. In this case, the eigenvalues are

$$
u \pm \sqrt{p^{\prime}(\rho)}
$$

For $t<1$, the equation $p^{\prime}(\rho)=0$ has exactly two roots in $[0,3]$, which we denote by $\rho_{\min }$ and $\rho_{\max }$. Therefore the system is strictly hyperbolic for $\rho<\rho_{\min }$ (vapor) and $\rho>\rho_{\max }$ (liquid), and is elliptic if $\rho_{\min }<\rho<\rho_{\max }$. Thus, the Cauchy problem for (1) with (2) is ill-posed. Nevertheless, the system can be regularised, with the viscous and capillarity terms of the Navier-Stokes-Korteweg system

$$
\left\{\begin{aligned}
\partial_{t} \rho+\operatorname{div}(\rho \mathbf{u}) & =0 \\
\partial_{t}(\rho \mathbf{u})+\operatorname{div}(\rho \mathbf{u} \otimes \mathbf{u}+p \mathcal{I}) & =\operatorname{div}(\boldsymbol{\tau})+\varepsilon \rho \nabla(\Delta \rho)
\end{aligned}\right.
$$

where $\varepsilon$ is the capillarity coefficient, and $\tau$ is the viscous tensor $\tau=$ $\mu\left(\nabla \mathbf{u}+\nabla^{T} \mathbf{u}\right)+\lambda \operatorname{div} \mathbf{\mathcal { I }}$, where $\mu$ and $\lambda$ are the viscosity coefficients. This allows to recover a solution to the Riemann problem when the initial left and right states are not in $\left[\rho_{\min }, \rho_{\max }\right]$, see $[6,9]$. 
The system (3) can be directly simulated [4]. Nevertheless, this requires to mesh the phase transition waves at the capillarity and viscosity scale, which can be very costly, especially if the flow is dominated by convection.

Other approaches for simulating (1) with (2) consist in the probabilistic Glimm scheme, and classical multicomponent algorithm (Volume-of-fluid, level-set and front tracking methods). The Glimm scheme, for which the final computed solution is an average of many probabilistic experiments, is very costly. The level-set methods has already been used in this context [7], but it results in a nonconservative scheme, which is ill-adapted for the approximation of nonlinear hyperbolic systems [3]. The main drawback of the front tracking and volume of fluid methods is the difficulty of their two dimensional implementation.

The basic problem, using a classical upwind method can be understood with a very simple example: suppose a Riemann problem with the two Maxwell states (see [7] for their definition): liquid on the left, and vapour on the right; both of the states have the same velocity $u$. The solution of such a problem is a moving phase transition at velocity $u$. With a classical upwind scheme, the discontinuity will diffuse, resulting in a density belonging to $\left[\rho_{\min }, \rho_{\max }\right]$, in which the system is no more hyperbolic.

The idea of the method we present relies on introducing a new variable, the volume fraction, which will be equal to 0 in the vapour, and 1 in the liquid. The aim is to cure the problem we just exposed, by putting the diffusion on the volume fraction, by keeping the density constant on the left and the right of the phase transition wave.

In the next section, we propose a diffuse interface formulation of the problem, in order to propose a diffuse interface numerical scheme.

\section{Reformulation as a multiphase sys- tem with source term}

In this section, we formulate (1) with (2) as a multiphase system with source term. The vapour and liquid phase are considered as two different fluids. We denote by $\chi_{i}(i=1,2)$ the indicator of the liquid and the vapour phase, i.e. if a phase is liquid, then $\chi_{1}=1$ and $\chi_{2}=0$, else $\chi_{1}=0$ and $\chi_{2}=1 . \chi_{i}$ follows an advection equation

$$
\partial_{t} \chi_{i}+\sigma \cdot \nabla \chi_{i}=0
$$

where $\sigma$ is the local phase transition velocity. Each phase follows

$$
\chi_{i}\left(\partial_{t} \mathbf{U}+\nabla \cdot F(\mathbf{U})\right)=0
$$


At the discrete level, $\chi$ will diffuse, so that it will belong to $[0,1]$. As in [2], we denote by $\mathbb{E}()$ an averaging operator. Taking the average of (4), and following [2] leads to

$$
\left\{\begin{array}{l}
\partial_{t} \mathbb{E}\left(\chi_{k} \mathbf{U}\right)+\nabla \cdot\left(\mathbb{E}\left(\chi_{k} F(\mathbf{U})\right)\right)=\mathbb{E}\left((F(\mathbf{U})-\sigma \mathbf{U}) \nabla \chi_{k}\right) \\
\partial_{t} \mathbb{E}\left(\chi_{k}\right)+\mathbb{E}\left(\sigma \cdot \nabla \chi_{k}\right)=0
\end{array}\right.
$$

We denote by $\alpha^{(k)}=\mathbb{E}\left(\chi_{k}\right)$. Note that system (6) is exactly the same as (1) when $\chi=0$ or $\chi=1$, and that the source term is localised only on the interfaces.

Let's go back to the example of Section 2 a moving discontinuity between the two Maxwell states. In the formulation (6), the aim is to put the numerical diffusion on $\alpha^{(k)}=\mathbb{E}\left(\chi_{k}\right)$, and to have a constant density on the left and on the right of the discontinuity.

\section{Solution of the Riemann problem}

Our numerical scheme will be based on a Godunov's method. Roughly speaking, the finite volume fluxes will be average of the fluxes resulting from the solution of heterogeneous Riemann problems, see Section 5 .

The full solution for the Riemann problem for (1) was derived in [7]. The first step is to compute the left (resp. right) wave curves, i.e. all the states that can be connected to a given state by a wave associated to the field $u-c$ (resp. $u+c$ ). Once these wave curves are computed, the solution of the Riemann problem is obtained by intersecting the left and the right wave curves.

The complexity of computing the wave curves is due to

- the fact that the fields on the vapour phase are not genuinely nonlinear;

- the phase transitions.

The non genuinely nonlinearity of the fields may induce composite waves. This means that a sonic wave may be not only a rarefaction wave or a shock, but also an attached wave (i.e. an undercompressive discontinuity followed by a rarefaction wave).

The occurrence of phase transition means that a sonic wave can be followed by a nonclassical shock [6]. Moreover, depending on the sound velocities on both sides of the phase transition, this nonclassical shock can be followed by another rarefaction wave.

To summarise, a left or right wave curve is composed of a shock wave or a rarefaction wave or an attached wave, which can be followed by a phase transition wave (nonclassical shock), which can itself be followed by a rarefaction wave in the vapour, provided the initial state is a liquid state.

Last, the solution of the Riemann problem is not unique. Detailing this problem is out of the scope of this paper, and we refer again 
to [7] for solving the problems of kinetic relation, and the nonuniqueness of the solution.

\section{$5 \quad$ Numerical scheme}

Our numerical scheme is derived from the original scheme of [1] which has already been extended to phase transition with a very different model in [5]. The computational domain is divided into geometrical entities, the cells, which can be, depending on the dimension, segments, triangle, quadrangle, hexaedra and tetrahedra. In each cell $K$, and for each fluid $i$, the unknowns are the cell average of the volume fraction $\alpha_{K}^{(i)}$, the density $\rho_{K}^{(i)}$ and the velocity $\mathbf{u}_{K}^{(i)}$. For simplicity, we will explain the numerical scheme in one dimension, because its extension to higher dimension is straightforward.

Following [1], each cell $k$ of the mesh is randomly filled with the fluid $i$, with probability $\alpha_{k}^{(i)}$, the events on different cells being independent. For one given realization, we integrate (5) for each fluid on the cell $k$, and between time $t^{n}$ and $t^{n+1}$. Then we perform an average on all the possible experience. We find

$$
\left(\alpha_{k}^{(i)} U_{k}^{(i)}\right)^{n+1}-\left(\alpha_{k}^{(i)} U_{k}^{(i)}\right)^{n}+\frac{\Delta t}{\Delta x}\left(\mathscr{F}_{k+1 / 2}^{(i)}-\mathscr{F}_{k-1 / 2}^{(i)}\right)=0
$$

where the fluxes $\mathscr{F}_{k+1 / 2}^{(i)}$ are the average of the integration of (5) computed for one given experience. On each cell interface, four cases may happen: liquid-liquid, liquid-vapour, vapour-vapour and vapour-liquid. Thus, this average can be written as

$$
\begin{aligned}
\mathscr{F}_{k+1 / 2}^{(i)}= & \mathscr{P}_{k+1 / 2}(l \mid l) \mathcal{F}_{k+1 / 2}^{(i)}\left(U_{k}^{(l)}, U_{k+1}^{(l)}\right) \\
& +\mathscr{P}_{k+1 / 2}(l \mid v) \mathcal{F}_{k+1 / 2}^{(i)}\left(U_{k}^{(l)}, U_{k+1}^{(g)}\right) \\
& +\mathscr{P}_{k+1 / 2}(v \mid v) \mathcal{F}_{k+1 / 2}^{(i)}\left(U_{k}^{(g)}, U_{k+1}^{(g)}\right) \\
& +\mathscr{P}_{k+1 / 2}(v \mid l) \mathcal{F}_{k+1 / 2}^{(i)}\left(U_{k}^{(g)}, U_{k+1}^{(l)}\right)
\end{aligned}
$$

where $\mathcal{F}_{k+1 / 2}^{(i)}\left(U_{k}^{\left(i_{1}\right)}, U_{k+1}^{\left(i_{2}\right)}\right)\left(i_{1}, i_{2}=v\right.$ or $\left.l\right)$ is the result of the integration of (5) for the Riemann problem between $U_{k}^{\left(i_{1}\right)}$ and $U_{k+1}^{\left(i_{2}\right)}$, and $\mathscr{P}_{k+1 / 2}\left(i_{1} \mid i_{2}\right)$ is the probability of the Riemann problem $\left(U_{k}^{\left(i_{1}\right)}, U_{k+1}^{\left(i_{2}\right)}\right)$ to happen. The computation of $\mathcal{F}_{k+1 / 2}^{(i)}\left(U_{k}^{\left(i_{1}\right)}, U_{k+1}^{\left(i_{2}\right)}\right)$, which is the integration of (5), strongly depends whether there is a jump of $\chi_{i}$ inside the cell or not. All the possible cases are listed in Figure 1, and explained in its caption.

For computing (7), it remains to compute the probabilities $\mathscr{P}$. We suppose that these probabilities depend only on the volume fraction on the left and right of the interface. Then, we go back to the example of Section 2. If we want to find the classical upwind finite volume scheme for the volume fraction, the only pos- 
sible choice is $\mathscr{P}_{k+1 / 2}(i \mid i)=\min \left(\alpha_{k}^{(i)}, \alpha_{k+1}^{(i)}\right)$ and $\mathscr{P}_{k+1 / 2}(i \mid j)=$ $\max \left(\alpha_{k+1}^{(i)}-\alpha_{k}^{(i)}, 0\right)$ if $i \neq j$, see [1] for more details.

The resulting numerical scheme has the property required at the end of Section 3: the densities of the liquid phase is always over $\rho_{\max }$, whereas the densities of the vapour phase always remain under $\rho_{\min }$. If we consider a phase transition wave between a liquid density $\rho_{\text {liq }}$, and a vapour density $\rho_{\text {vap }}$, a classical upwind scheme would diffuse the density, resulting in a density in the convex hull of $\left\{\rho_{\text {liq }}, \rho_{\text {vap }}\right\}$, so that it would belong to $\left[\rho_{\min }, \rho_{\max }\right]$, in which the system is elliptic. Contrarily, this scheme will keep the liquid and vapour densities constant, and will diffuse the volume fraction $\alpha$, which will belong to $[0,1]$. Of course, the average density $\alpha^{(1)} \rho^{(1)}+\alpha^{(2)} \rho^{(2)}$ can belong to $\left[\rho_{\min } ; \rho_{\max }\right]$, but this average density is never used in the numerical scheme.

\section{$6 \quad$ Numerical Tests}

All the numerical tests are performed with the adimensioned temperature of (2) equal to 0.8 . The kinetic relation that was practically implemented is the same as in [7]. This choice allows to solve the Riemann problem and to define a local velocity of phase transition $\sigma$.

\subsection{Riemann problem}

In this first numerical test, we compute the solution of the same Riemann problem as [7], which initial solutions are summarised in the following table

\begin{tabular}{|c|c|c|c|c|}
\hline$\rho_{l}$ & $u_{l}$ & $x_{0}$ & $\rho_{r}$ & $u_{r}$ \\
\hline$\rho_{\text {liquid }}^{M}=1.28943$ & -3.5 & 0. & $\rho_{\text {vapour }}^{M}=0.726691$ & 0. \\
\hline
\end{tabular}

The computational domain is $[-1.5 ; 0.5]$, and the solution is computed until $t=0.3$. The computed solutions are shown on Figure 2, and show the convergence towards the analytical solution.

\subsection{Collapse of a metastable vapour in a liq- uid}

In this numerical test, we perform a two dimensional computation. The initial condition consists in a two dimensional bubble of metastable vapour $(\rho=0.835)$, with radius 0.5 inside a liquid $(\rho=1.31)$. The computational domain is the square $[-1 ; 1]^{2}$, with the boundary condition $\partial \mathbf{F} / \partial \mathbf{n}=0$, where $\mathbf{n}$ is the external normal of the boundary. The numerical results are shown on Figure 3. 


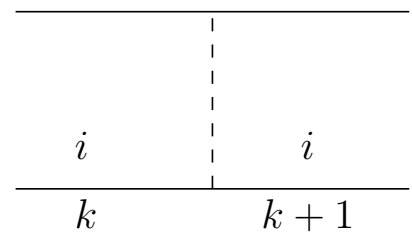

(a)

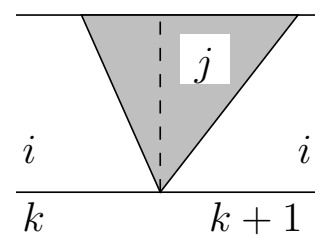

(c)

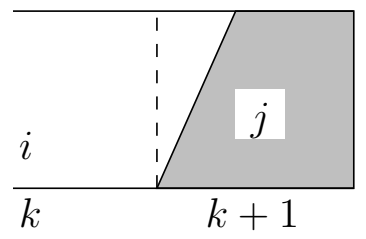

(e)

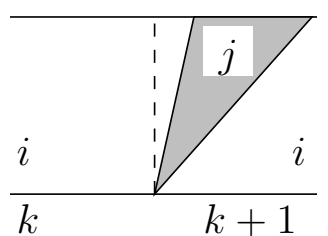

(b)

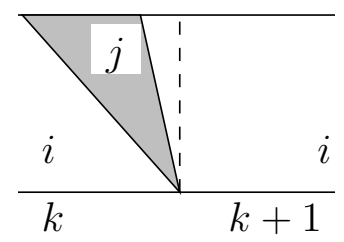

$(d)$

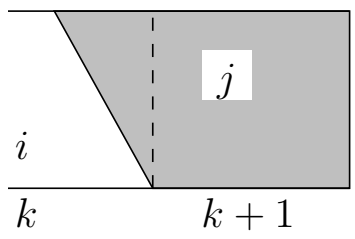

$(f)$

Figure 1: Discontinuities of $\chi$ depending on the solution of the Riemann problem. Integration of (5) depends on the number and the position of interfaces between the fluid $i$ and the fluid $j$ that are inside the cell. These six cases can be gathered into three cases, depending on the position of the interfaces. These three cases are listed from the easiest to the most difficult. Cases $(a),(b)$ and $(e)$ do not have any interface inside the cell $k$. Integration gives an Eulerian flux on the fluid $i$, as in the classical finite volume method, i.e. $F(U)$ where $U$ is the value on the boundary of the cell. In the cases, $(c)$ and $(f)$, it is needed to integrate of one interface inside the cell, so that the integration gives one transfer flux on the fluid $i$ and $j$, and one Eulerian flux on the fluid $j$. A transfer flux is defined as $F(U)-\sigma U$ where $U$ is the value on the left or on the right side of the discontinuity (they are equal because the Rankine-Hugoniot relations hold). In the case $(d)$, it is needed to integrate two interfaces inside the cell. This results in two transfer fluxes on each fluid, and one Eulerian flux on the fluid $i$. 


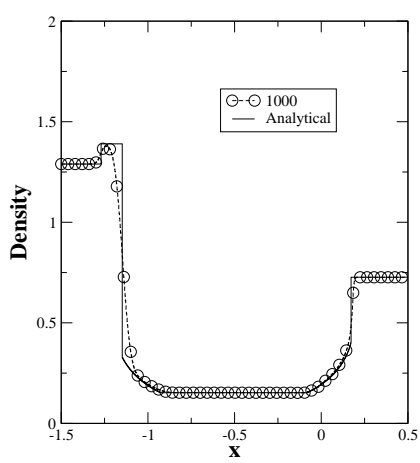

(a)

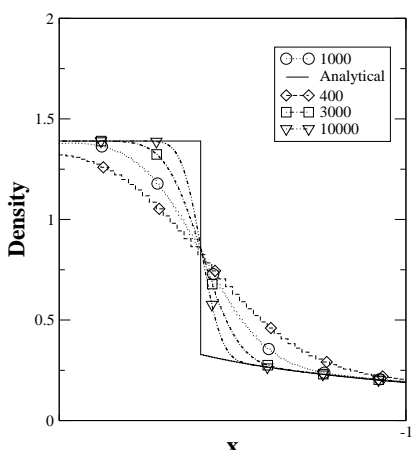

(c)

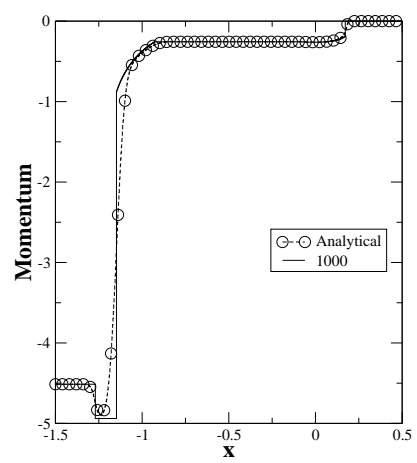

(b)

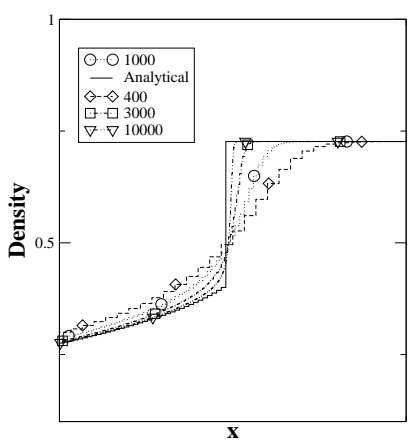

$(d)$

Figure 2: Numerical results for the test of the Riemann problem. The left wave is a shock, followed by a phase transition wave, followed by a rarefaction wave, whereas the right wave is an attached wave, i.e. a discontinuity followed by a rarefaction wave. The numerical solution for a mesh of 1000 points is compared with the analytical one: the average density on Figure $(a)$, and the momentum on Figure $(b)$. We then perform a convergence test for the average density: the numerical solution is computed with a mesh of 400, 1000, 3000 and 10000 points, and we show the zoom of the results on each of the waves: $(a)$ for the left wave, and $(b)$ for the right wave. 


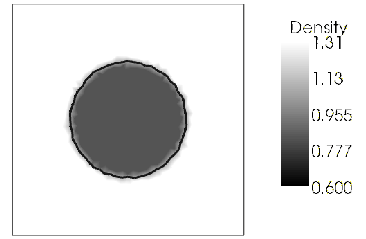

(a)

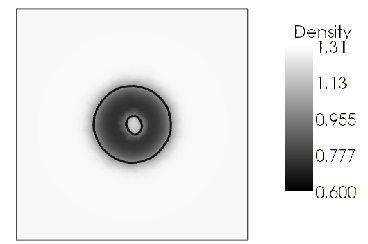

$(c)$

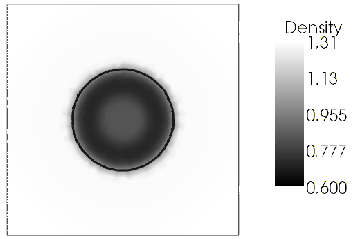

(b)

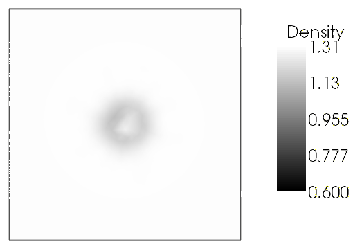

$(d)$

Figure 3: Numerical results for the test of the collapse of a bubble of metastable vapour in a liquid. We show a grey-scale of the average density, and the isovalue $\alpha=0.5$ as a black line, which is the limit between the liquid and the vapour. At initial time, see Figure $(a)$, the bubble of metastable vapour is inside the vapour. Its metastability induces a phase transition, but also a shock inside the bubble, see Figure $(b)$. The shock focalises inside the bubble. The reflection of the shock inside the bubble induces a phase transition, so that a droplet appears inside the bubble, see Figure (c). Finally, the bubble collapses when the two phase transition waves encounters, see Figure $(d)$. 


\section{Conclusion}

A numerical method for the simulation of the isothermal Euler system with van-der-Waals equation was derived and tested with numerical tests. The main plus of this method, compared with previous numerical schemes is that it is fully conservative. The two dimensional version of the scheme is easy, and treating cavitation or nucleation is straightforward.

The main drawback of the method is that it is a diffuse interface method. Having a sharper interface can be achieved by extending the numerical scheme to higher order. A discontinuous Galerkin version of this method was presented at the conference, nevertheless, it was too long to expose it here, and this will be the object of a forthcoming paper.

\section{References}

[1] Rémi Abgrall and Richard Saurel. Discrete equations for physical and numerical compressible multiphase mixtures. J. Comput. Phys., 186(2):361-396, 2003.

[2] Donald A. Drew and Stephen L. Passman. Theory of multicomponent fluids, volume 135 of Applied Mathematical Sciences. Springer-Verlag, New York, 1999.

[3] Thomas Y. Hou and Philippe G. LeFloch. Why nonconservative schemes converge to wrong solutions: error analysis. Math. Comp., 62(206):497-530, 1994.

[4] D. Jamet, O. Lebaigue, N. Coutris, and J. M. Delhaye. The second gradient method for the direct numerical simulation of liquidvapor flows with phase change. J. Comput. Phys., 169(2):624$651,2001$.

[5] Olivier Le Métayer, Jacques Massoni, and Richard Saurel. Modelling evaporation fronts with reactive Riemann solvers. J. Comput. Phys., 205(2):567-610, 2005.

[6] Philippe LeFloch. Propagating phase boundaries: formulation of the problem and existence via the Glimm method. Arch. Rational Mech. Anal., 123(2):153-197, 1993.

[7] Christian Merkle and Christian Rohde. The sharp-interface approach for fluids with phase change: Riemann problems and ghost fluid techniques. M2AN Math. Model. Numer. Anal., 41(6):1089-1123, 2007.

[8] Denis Serre. Systems of conservation laws (1 \& 2). Cambridge University Press, Cambridge, 1999.

[9] M. Slemrod. Admissibility criteria for propagating phase boundaries in a van der Waals fluid. Arch. Rational Mech. Anal., 81(4):301-315, 1983. 